\title{
Spatial Dependence of the Nonlinear BOLD Response at Short Stimulus Duration
}

\author{
J. Pfeuffer, J.C. McCullough, P.-F. van de Moortele, K. Ugurbil, X. Hu \\ Center for Magnetic Resonance Research, University of Minnesota Medical School, Minneapolis, MN, USA
}

Non-linearities in the measured BOLD data were found for the response integral and width. With the high sensitivity at $7 \mathrm{~T}$, a voxel-based analysis was feasible and was used to investigate the spatial dependence of the non-linearities at high spatial resolution. Correlation between response onset and non-linearity suggests that the origin of the observations are a tissue-specific phenomenon. Comparison with $4 \mathrm{~T}$ and $1.5 \mathrm{~T}$ data also indicates a field dependence. The observed non-linearity at short stimulus duration (SD) has likely a considerable hemodynamic contribution and thus must be accounted for in event-related fMRI paradigms.

Introduction Most fMRI studies use linear models to predict the measured response by convolution of the impulse response with the stimulus profile [1]. Using short stimuli, non-linear effects were reported for amplitude and width [e.g.,1-3]. In this study, high temporal and spatial resolution was used to explore non-linear effects and investigate their spatial dependence.

Methods Single-shot EPI with a voxel size of $1.2 \times 1.2 \times 2 \mathrm{~mm}^{3}$ was performed on a $7 \mathrm{~T} / 90 \mathrm{~cm}$ system using a $7-\mathrm{cm}$ quadrature T/R coil pair (TE $20 \mathrm{~ms}$, TR $125 \mathrm{~ms}$, sagittal plane). With a zoomed imaging technique, BISTRO [4], the FOV was reduced to $4.8 \times 15.4 \mathrm{~cm}^{2}$. Event-related fMRI studies were performed on four volunteers (visual stimuli with LED goggles at $8 \mathrm{~Hz}$ ). With interstimulus intervals fixed at $18 \mathrm{~s}$, stimulus duration (SD) took values of $0.25,0.5,1$, and $2 \mathrm{~s}$. 16 trials were executed per series $(5 \mathrm{~min}$ ). The integral and width of the BOLD response were determined with single-voxel analysis. For both parameters, a non-linearity index $\left(\mathrm{NL}^{\mathrm{INT}}\right.$ and $\left.\mathrm{NL}^{\mathrm{WIDTH}}\right)$ was defined as the normalized difference $\left[\mathrm{R}^{\exp }(\mathrm{SD}=0 \mathrm{~s})-\mathrm{R}^{\text {theo }}(\mathrm{SD}=0 \mathrm{~s})\right] / \mathrm{R}^{\exp }(\mathrm{SD}=2 \mathrm{~s})$ between experimental and theoretical values, obtained by extrapolation to $\mathrm{SD}=0$ s. Also, a corresponding $\mathrm{NL}^{\mathrm{INT}}$ map was computed. Cross correlation was used to determine activation maps and onset lag.

$\underline{\text { Results }}$ Non-linearities were observed for the amplitude and the integral of the BOLD response (positive component). The deviation from the linear model increased with decreasing SD (Fig. 1). NL ${ }^{\text {INT }}$ was determined from the intercept of a linear fit and found to be $(8 \pm 2) \%$ (4 subjects) for the averaged time course (voxel and epoch average). Comparable experiments at $4 \mathrm{~T}$ using $3.1 \times 3.1 \times 6 \mathrm{~mm}^{3}$ resolution [3], revealed a significantly higher $\mathrm{NL}^{\mathrm{INT}}$ of $(30 \pm 3) \%$ (5 subjects). A motor study at $1.5 \mathrm{~T}$ reported a $\mathrm{NL}^{\text {INT }}$ of $40 \%$ [1]. These observations indicate a magnetic field dependence of the non-linearity in the response integral.

Previously reported non-linearities in the width (study at 4T [3]) were also observed in the present study at 7T using higher spatial resolution. NL ${ }^{\text {WIDTH }}$ was $-31 \%$ (Fig. 2), indicating that the FWHM at short SD is much smaller than expected from a linear model (simulated curve in Fig. 2).

Maps of $\mathrm{NL}^{\mathrm{INT}}$ and onset time are shown in Fig. 3a, 3b, demonstrating that the NL is clustered in patterns that are virtually identical to those in the onset map. With the spatial non-linearity information resolved, NL ${ }^{\text {INT }}$ was found to be up to $40 \%$. Most important, significant linear correlation between $\mathrm{NL}^{\mathrm{INT}}$ and onset was found (CC-weighted linear fit). Responses with a later onset revealed less non-linearity.

In summary, a significant spatial dependence of non-linearities was found, which is hypothesized to be tissue-type specific. Signal-to-noise gains at $7 \mathrm{~T}$ provided sufficient sensitivity for simultaneously high temporal and high spatial resolution. The dependence of $\mathrm{NL}^{\mathrm{INT}}$ on the magnetic field and the demonstrated non-linearity in the width supports the argument that the source of the non-linearity at short SD is not only of neuronal origin, but has also a considerable hemodynamic contribution. Understanding the non-linearities in the BOLD response is significant for modeling event-related and single-trial fMRI experiments.
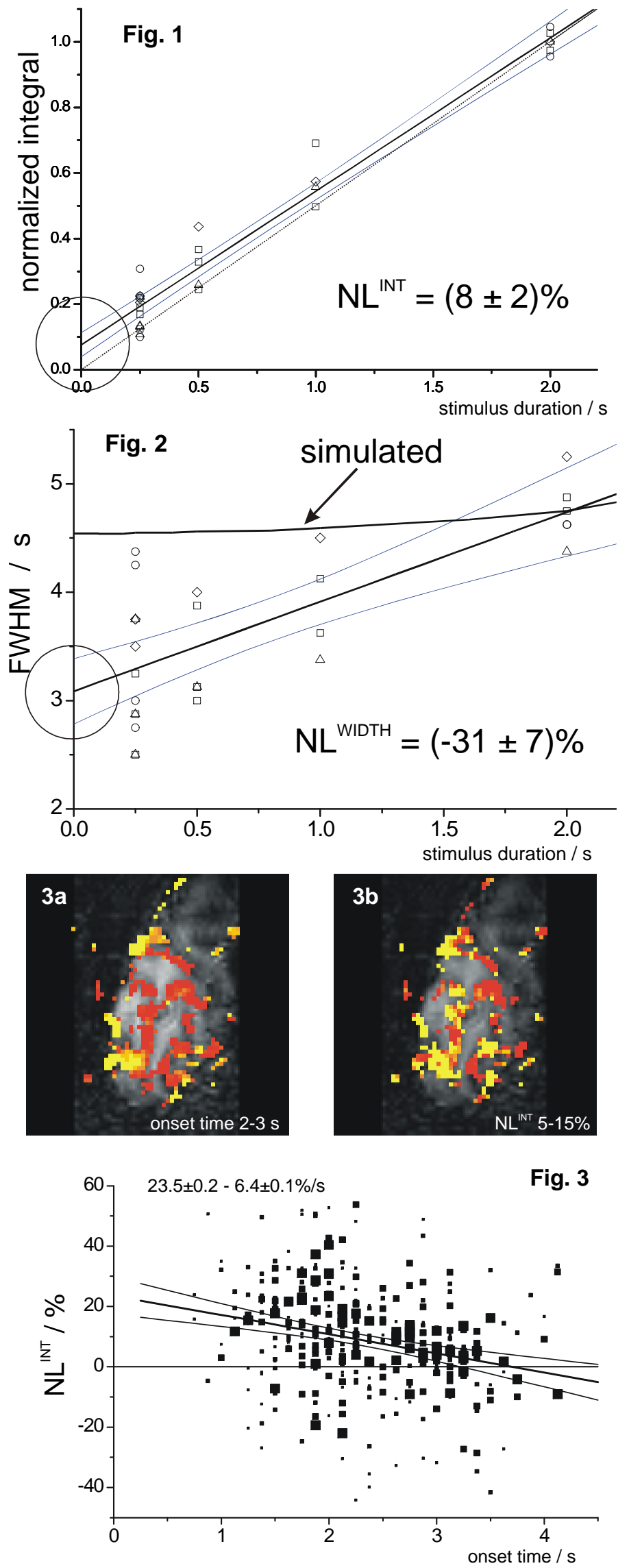

References 1. Glover GH Neuroimage 9:416;1999. 2. Vazquez AL Neuroimage 7:108;1998. 3. Pfeuffer J ISMRM 988;2000. 4. Garwood M, MRM 1095;2001. Supported by NIH RR08079, RR09784, MH55346, W. M. Keck Fdn, MIND Inst. 